\title{
Geometric indexes of heart rate of variability identifies autonomic alterations in young patients with type 1 diabetes mellitus
}

\author{
Naiara Maria Souza MSc, Calos Marcelo Pastre PhD, Anne Kastelianne França da Silva MSc, \\ Aline Fernanda Barbosa Bernardo MSc, Franciele Marques Vanderlei PhD, Talys Naomi Harada Bonora, \\ Francis Lopes Pacagnelli PhD, Gustavo Carvalho Machado PhD, Luiz Carlos Marques Vanderlei PhD
}

NM Souza, CM Pastre, AK França da Silva, et al. Geometric indexes of heart rate of variability identified autonomic alterations in young patients with type 1 diabetes mellitus. Curr Res Cardiol 2016;3(2):38-42.

OBJECTIVES: To compare autonomic modulation in young patients with type 1 diabetes mellitus (DM1) $(n=20)$ and healthy control subjects $(n=23)$ using geometric indexes of heart rate variability (HRV) associated with linear domains of time and frequency; and to verify correlations between geometric indexes and those obtained by the time and the frequency domain of diabetic individuals.

METHODS: Heart rate was recorded as beat-to-beat with subjects supine for $30 \mathrm{~min}$. The following indexes were calculated: RRtri, TINN, Poincaré plot, SD1, SD2, SD1/SD2, RMSSD, SDNN, LF and HF (ms²/un) and LF/HF. Visual analysis of the Poincaré plot was also performed. Independent Student's $t$ test or Mann-Whitney test, and Spearman's correlation test or Pearson's correlation test were used for statistical analysis .

RESULTS: Significantly lower values of SD1, SD2, RRtri, RMSSD, SDNN, and LF and HF in $\mathrm{ms}^{2}$ were observed in patients with DM1 compared with healthy subjects.

CONCLUSION: The Poincaré plot showed less dispersion of RR intervals for the DM1 group. Strong correlations were observed with the geometric indexes with the time domain and frequency indexes.

Key Words: Autonomic nervous system; Diabetes mellitus type 1; Heart rate; Nonlinear dynamics; Young
Cardiovascular autonomic neuropathy (CAN) is one of the most

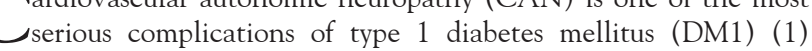
because it affects the fibres of the autonomic nerves of the heart and blood vessels, causing heart rate abnormalities and vascular dynamic impairments $(2,3)$, leading to increased mortality $(4,5)$.

Changes in the autonomic modulation of DM1 were previously described through heart rate variability (HRV) (6-9), a noninvasive assessment method that measures the oscillation of intervals between consecutive heart beats (RR), which are related to influences from the autonomic nervous system (ANS) on the sinus node $(10,11)$.

Most studies that assessed autonomic modulation by HRV included populations with type 2 diabetes mellitus $(9,12-15)$, a population that normally have associated comorbidities that could interfere in autonomic modulation (12). Also, these studies used linear methods of analysis of frequency and time domains $(14,15)$, which present limitations as stationary requirements, and show more sensitivity to interference and ectopic beats when compared with nonlinear methods (16). Nonlinear methods are based on chaos theory and may enable better discrimination between normal and abnormal ANS physiology (6). For example, the Poincaré plot is a method used for geometric and nonlinear analysis of the autonomic modulation (16-18). A previous study involving type 2 diabetic individuals assessed different methods of HRV and reported that geometric methods are more reliable for evaluating the presence of an autonomic diabetic disease (19), and studies that analyze this type of indexes in DM1 population were not deeply explored.

Therefore, geometric methods could identify autonomic changes and help to plan a better treatment strategy in DM1 patients. Thus, the aim of the present study was: to compare HRV indexes obtained by geometric methods (RRtri, TINN and Poincaré plot - SD1, SD2 and SD1/SD2), associated to indexes of time domain (RMSSD and SDNN) and frequency domain (LF, HF ( $\left.\mathrm{ms}^{2} / \mathrm{un}\right)$ and LF/HF), of individuals with DM1 and healthy control subjects; and to verify correlations between geometric indexes and those obtained by the time and the frequency domain of diabetic individuals.

\section{Subjects}

\section{METHODS}

The present study included 43 young adults who were divided into two groups: the DM1 group ( $\mathrm{n}=20$ [nine men, 11 women]) with a mean $( \pm$ SD) age $22.05 \pm 4.35$ years, with $10.60 \pm 4.81$ years of DM1 diagnosis, and a control group with 23 healthy subjects (15 men, eight women) mean age $21.69 \pm 2.42$ years.

The inclusion criteria were absence of cardiorespiratory diseases and no use of medication that could interfere with heart autonomic activity. For the DM1 group, one other criteria that was analyzed was that the individual had to have a medical diagnosis of DM1. Smokers and alcoholics were excluded from the present study. Those who met the inclusion criteria were given an explanation of the aims and procedures of the study and signed an informed consent form. All of the procedures used in the present study were approved by the research ethics committee of the authors' university (Protocol No. 47/2011).

\section{Study design}

The present study was an open, cross-sectional, nonrandomized analysis. The study protocol did not allow influence from evaluators or reviewers.

Universidade Estadual Paulista "Julio de Mesquita Filho", Faculdade de Ciências e Tecnologia.

Correspondence: Ms Naiara Maria Souza, Department of Physiotherapy, Universidade Estadual Paulista - FCT/UNESP, Roberto Simonsen, 305,

Presidente Prudente, São Paulo, Brazil, 19060-900. Telephone 55-18-32214391, fax 55-18-3221-4391, e-mail naiara_bs@live.com 
The present study consisted of three parts: identification, physical evaluation and autonomic evaluation. During the identification, subjects were assessed whether they met the inclusion criteria, and demographic data information were collected to characterize the population. Following identification, physical (anthropometric measurements, heart rate and blood pressure at rest) and autonomic evaluations were performed. The assessments were conducted in a controlled, quiet room with a temperature of $23.87 \pm 2.54^{\circ} \mathrm{C}$ and humidity of $54.22 \pm 8.51 \%$. All evaluations were performed in the afternoon (between 13:00 and 17:00).

\section{Identification}

Initially, subjects were characterized using questions with regard to age, sex, signs and symptoms of diabetes, use of drug therapy, presence of comorbidities, smoking and alcohol intake, and the level of physical activity measured using the International Physical Activity Questionnaire (20).

\section{Physical evaluation}

Anthropometric measures were collected to calculate body mass index (BMI), and waist and hip circumferences to obtain the waist/hip $(\mathrm{W} / \mathrm{H})$ ratio $(21,22)$. The assessment of heart rate was made by palpation of the brachial artery for $1 \mathrm{~min}$, and blood pressure was indirectly measured using a stethoscope (Littman, USA) and an aneroid sphygmomanometer (Welch Allyn-Tycos, USA) on the left arm of the subjects as recommended by the VI Brazilian Guidelines on Hypertension (23).

\section{Autonomic evaluation}

Evaluations of HRV were performed to verify autonomic modulation. Subjects were instructed to avoid alcoholic and/or ANS stimulants such as coffee, tea or chocolate for a period of $24 \mathrm{~h}$ before the assessment. During the autonomic evaluation, the participants were instructed to remain alert, quiet, with spontaneous rest breathing in supine for $30 \mathrm{~min}$ on a couch. After receiving the instructions, an electrode was placed on the participant's chest at the sternal angle level using an elastic strap, and the heart rate receiver (Electro S810i, Polar, Finland) was attached to the patient's wrist. This equipment has been previously validated to record beat-to-beat heart rate for collecting HRV data $(24,25)$.

To analyze HRV indexes, 1000 intervals of consecutive cardiac beats were used, selected after digital filtering, complemented by manual filtering to eliminate artifact and ectopic beats, and only series with $>95.0 \%$ of sinus beats were included in the analysis (26).

\section{Analysis of indexes of HRV}

HRV analysis was performed using geometric methods (RRtri, TINN and Poincaré plot - SD1, SD2 and SD1/SD2) in addition to indexes of time domain (SDNN and RMSSD) and frequency domain (LF, HF [ms²/un] and LF/HF). The RRtri and TINN indexes were calculated from the construction of the density histogram of the normal RR, which all possible values of RR are shown on the horizontal axis $(\mathrm{x}$-axis) and on the vertical axis (y-axis) the frequency in which they occurred (17). The RRtri index is the integral of the histogram (total number of RR) divided by the maximum density distribution (the modal frequency of RR), measured on a discrete scale with boxes of $7.8125 \mathrm{~ms}(1 / 128 \mathrm{~s})$, while the triangular interpolation interval histogram of RR (TINN) is the baseline width of the distribution, measured as the base of the triangle, with the error of least squares used to determine the triangle (27).

The Poincaré plot is a two-dimensional graphic and represents the correlation between consecutive RR, in which each interval is plotted against the next interval forming a figure that enables the extraction of SD1, SD2 and SD1/SD2 ratio, beyond the qualitative analysis of its attractor (28).

The SD1 index represents the dispersion of perpendicular points to the line of identity, and is considered to be an index of instantaneous recording of beat-to-beat variability (ie, representative of the parasympathetic modulation activity). The SD2 index represents the dispersion of points along the identity line and represents the variability in long-term records; thus, this index is representative of the conjunct action of the sympathetic and parasympathetic modulation activities. The SD1/SD2 ratio is the ratio between the short- and long-term fluctuations of the RR intervals (10).

The qualitative analysis of Poincaré plot was made by figures that were formed by its attractor, as described by Tulppo et al (29): a picture with an increment of the RR dispersion, characteristic of a normal plot; and a figure with small RR dispersion characteristic of a plot with less variability. A single evaluator analyzed all figures.

For the time domain, the RMSSD and SDNN indexes were calculated. The RMSSD is the square root of the mean squared differences between adjacent normal RR and the SDNN index is a standard deviation of all normal RR, recorded in a time interval $(10,13)$. Analyses in the frequency domain were calculated using the algorithm of fast Fourier transform. The spectral components of low frequency (LF: $0.04 \mathrm{~Hz}$ to $0.15 \mathrm{~Hz}$ ), high frequency (HF: $0.15 \mathrm{~Hz}$ to $0.40 \mathrm{~Hz}$ ) and the ratio between these components ( $\mathrm{LF} / \mathrm{HF}$ ratio) were used and expressed in $\mathrm{ms}^{2}$ and in normalized units (nu) (10).

The analysis was processed using computer software (Kubios, Version 2.0, Biosignal Analysis and Medical Image Group, Department of Physics, University of Kuopio, Finland) (30).

\section{Statistical analysis}

Initially, data distribution was determined using the Shapiro-Wilk test. For variables with normal distribution, the independent Student's $t$ test was used (body mass, height, W/H, SBP, DBP, HR, LF and HF [nu], TINN and SD1/SD2). For variables without normal distribution (BMI, age, LF and HF [ms²], LF/HF, RMSSD, SDNN, RRtri, SD1 and SD2) Mann-Whitney test was used. Correlations between the geometric indexes and time and frequency domains in the DM1 group were analyzed using Pearson's correlation test when data had a normal distribution (LF and HF [nu], TINN and SD1/SD2) or by Spearman's correlation test when normal distribution was not found (SDNN, RMSSD, LF $\left[\mathrm{ms}^{2}\right], \mathrm{HF}\left[\mathrm{ms}^{2}\right], \mathrm{LF} / \mathrm{HF}, \mathrm{RR}$ tri, SD1 and SD2). The correlation coefficients $(r)$ were classified as moderate when the values were between 0.60 and 0.79 , or as excellent when the values were $>0.80$ (31). The level of statistical significance was set at $5 \%$.

\section{RESULTS}

The characteristics of the DM1 and control groups are summarized in Table 1 . There were no statistically significant differences between groups, confirming homogeneity between the groups. All subjects from the DM1 group were insulin dependent, four (20\%) were using medication to control blood pressure, one $(5 \%)$ was using medication for thyroid disorders, two (10\%) were using medication for peripheral neuropathy symptoms and two (10\%) were using contraceptive. Regarding comorbidities, each group had two individuals classified as grade I obesity (21).

Table 2 shows the HRV indexes values of time and frequency domains. Reduced values of the LF and HF $\left(\mathrm{ms}^{2}\right)$, RMSSD and SDNN indexes were found in the DM1 when compared with the control group.

The geometric indexes of HRV values are reported in Table 3. The RRtri, SD1 and SD2 indexes values were lower for the subjects in the DM1 group compared with the control group.

Figure 1 shows the Poincaré plot of one subject in each group. These figures were chosen based on the subjects that showed SD1 and SD2 values that were similar to the average of their respective group. It is observed less dispersion of points for the plot in the subject from the DM1 group compared with the control group subjects.

Table 4 presents the correlations values between geometric indexes and time and frequency indexes of DM1 subjects. There are excellent correlations of geometric indexes with the indexes obtained by the time and frequency domains. 
TABLE 1

Characteristics of participants in the control and diabetic (DM1) groups

\begin{tabular}{lccc}
\hline Variable & Control $(\mathbf{n}=\mathbf{2 3})$ & DM1 $(\mathbf{n}=\mathbf{2 0})$ & $\mathbf{P}$ \\
\hline Age, years & $21.69 \pm 2.42(19-29)$ & $22.5 \pm 4.35(18-30)$ & 0.6004 \\
Body mass, kg & $72.68 \pm 14.69$ & $71.73 \pm 11.24$ & 0.8147 \\
& $(50.00-100.00)$ & $(56.30-93.80)$ & \\
Height, $\mathrm{m}$ & $1.73 \pm 0.11(1.51-1.93)$ & $1.69 \pm 0.10(1.54-1.93)$ & 0.1881 \\
$\mathrm{BMI}, \mathrm{kg} / \mathrm{m}^{2}$ & $23.96 \pm 3.70(17.8-31.9)$ & $25.03 \pm 3.48(21.3-34.3)$ & 0.3362 \\
W/H & $0.79 \pm 0.09(0.60-0.93)$ & $0.77 \pm 0.05(0.67-0.87)$ & 0.5919 \\
HR, beats $/ \mathrm{min}$ & $75.56 \pm 10.61(60-92)$ & $80.20 \pm 10.52(64-97)$ & 0.1592 \\
SBP, mmHg & $114.52 \pm 11.65(90-140)$ & $109.00 \pm 11.19(90-130)$ & 0.1221 \\
DBP, mmHg & $73.91 \pm 9.40(60-100)$ & $68.50 \pm 9.88(50-80)$ & 0.0733 \\
IPAQ, min & $790.00 \pm 761.36(60-2990)$ & $545.60 \pm 679.53(0-3080)$ & 0.2189 \\
\hline Dat presen
\end{tabular}

Data presented as mean $\pm S D$ (minimum - maximum). BMI Body mass índex; HR Heart rate; SBP Systolic blood pressure; DBP Diastolic blood pressure; DM1 Type 1 diabetes mellitus; IPAQ Physical activities per week; W/H Waistto-hip ratio

TABLE 2

Values of heart rate variability indexes in the frequency (LF, LF, HF, HF and LF/HF) and time (RMSSD and SDNN) domains

\begin{tabular}{|c|c|c|c|}
\hline Index & Control & DM1 & $\mathbf{P}$ \\
\hline LF, un & $48.03 \pm 16.57$ & $52.39 \pm 15.37$ & 0.3789 \\
\hline$L F, \mathrm{~ms}^{2}$ & $1269.9 \pm 760.19$ & $894.00 \pm 1238.6$ & 0.0077 \\
\hline HF, un & $51.96 \pm 16.57$ & $47.60 \pm 15.37$ & 0.3789 \\
\hline $\mathrm{HF}, \mathrm{ms}^{2}$ & $1446.5 \pm 947.48$ & $901.65 \pm 1220.8$ & 0.0036 \\
\hline LF/HF & $1.21 \pm 1.09$ & $1.40 \pm 1.06$ & 0.5838 \\
\hline RMSSD, ms & $60.75 \pm 22.70$ & $40.9 \pm 27.91$ & 0.0038 \\
\hline SDNN, ms & $72.45 \pm 19.99$ & $46.08 \pm 26.45$ & 0.0004 \\
\hline
\end{tabular}

Data presented as mean \pm SD. DM1 Type 1 diabetes mellitus; HF Highfrequency component; LF Low-frequency component; LF/HF Ratio of low-frequency and high-frequency components; RMSSD Square root of the mean squared differences between adjacent normal RR intervals; SDNN SD of all normal RR intervals; un Standard unit

\section{DISCUSSION}

The results of the present study showed lower values of geometric indexes and a decrease in the linear indexes of the time and frequency domain in DM1. Therefore, such values of HRV indexes suggest a reduced autonomic modulation for sympathetic and parasympathetic modulation activities.

Geometric indexes RRtri and SD2, which reflect global HRV, showed lower values for the DM1 group when compared with the control group, suggesting that the DM1 promotes reduction in ANS global activity. This feature is indicative of abnormal and insufficient adaptation of this system, and could cause impairment in physiological functioning of all organs mediated by the ANS (10). Furthermore, decreases in the global HRV could be considered as an early manifestation of a cardiac autonomic nerve damage, a subclinical presentation of CAN, which increases the risk for arrhythmia, myocardial ischemia and sudden death in this population (4).

The TINN index represents the ANS global activity and, although it was shown to be lower in the DM1 group, this result was not statistically significant.

The literature presents conflicting findings with regard to geometrical indexes. May et al (32) found no statistically significant differences for RRtri index among individuals with diabetes mellitus type 2, 40 to 75 years of age and healthy subjects, whereas Seyd et al (9) reported lower values of TINN and RRtri indexes for diabetic subjects, indicating reduced overall HRV for 70 subjects with a diagnosis of noninsulin-dependent diabetes mellitus and 64 healthy volunteers.

The SD1 index, which represents parasympathetic autonomic modulation $(17,28,33)$, was lower in individuals with DM1 when
TABLE 3

Values of heart rate variability of geometric indexes

\begin{tabular}{lccc}
\hline Index & Control & DM1 & P \\
\hline RRtri, S & $18.02 \pm 4.12$ & $12.81 \pm 6.71$ & 0.0009 \\
TINN, ms & $218.26 \pm 82.78$ & $203.50 \pm 98.69$ & 0.2424 \\
SD1, ms & $42.99 \pm 16.05$ & $28.98 \pm 19.76$ & 0.0038 \\
SD2, ms & $92.21 \pm 26.29$ & $58.00 \pm 32.47$ & 0.0002 \\
SD1/SD2 & $0.47 \pm 0.14$ & $0.47 \pm 0.13$ & 0.9554 \\
\hline
\end{tabular}

Data presented as mean \pm SD. DM1 Type 1 diabetes mellitus; SD2 SD of long-term of continuous RR intervals; SD1 SD of the variability of instantaneous beat to beat; SD1/SD2 Ratio of SD1 to SD2; RRtri Triangular index; TINN Interpolating triangular histogram of RR intervals

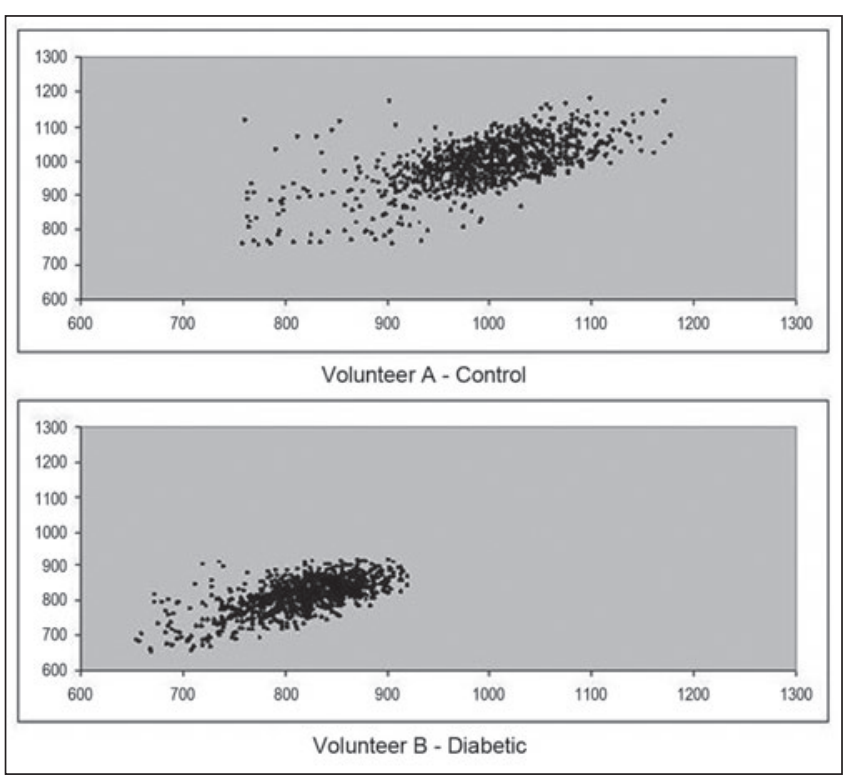

Figure 1) Visual standard of Poincare plot observed in the control (volunteer $A-S D 1=42.4$ and $S D 2=88.7)$ and DM1 group (volunteer $B-$ $\mathrm{SD} 1=27.8$ and SD2 $=60.9) \cdot R R(n)(m s)$ on the $x$-axis and $R R(n+1)$ (ms) on the $y$-axis

\section{TABLE 4}

Correlation coefficient ( $r$ ) of geometric indexes and the time and frequency domains in the type 1 diabetes mellitus group

\begin{tabular}{|c|c|c|c|c|c|}
\hline Index & RRtri, s & TINN, ms & SD1, ms & $\mathrm{SD} 2, \mathrm{~ms}$ & SD1/SD2 \\
\hline SDNN, ms & $0.9210^{\star \star}$ & $0.9465^{\star \star}$ & $0.9395^{\star \star}$ & $0.9872^{\star \star}$ & $0.5288^{*}$ \\
\hline RMSSD, ms & $0.8736^{\star \star}$ & $0.8814^{\star \star}$ & $1.000^{\star *}$ & $0.8876^{\star \star}$ & 0.7579 ** \\
\hline $\mathrm{LF}, \mathrm{ms}^{2}$ & $0.8774^{* \star}$ & $0.8625^{\star *}$ & $0.8030^{\star *}$ & $0.9695^{\star *}$ & 0.3083 \\
\hline $\mathrm{HF}, \mathrm{ms}^{2}$ & $0.9368^{\star \star}$ & $0.9032^{\star \star}$ & $0.9549 * *$ & $0.9124^{\star \star}$ & $0.6602^{*}$ \\
\hline LF/HF & -0.3278 & -0.2622 & $-0.4957^{*}$ & -0.1843 & $-0.8334^{* *}$ \\
\hline LF, un & -0.3278 & -0.2205 & $-0.4957^{*}$ & -0.1843 & $-0.8151^{* *}$ \\
\hline HF, un & 0.3278 & 0.2205 & $0.4957^{*}$ & 0.1843 & $0.8151^{\text {** }}$ \\
\hline
\end{tabular}

${ }^{*} P<0.05 ;{ }^{*} P<0.0001$. HF High-frequency component; LF Low-frequency component; LF/HF Ratio of LF to HF; RRtri Triangular index; SD1 SD of the variability of instantaneous beat-to-beat; SD2 SD of the long-term continuous RR intervals; SD1/SD2 Ratio of SD1 to SD2; SDNN SD of all normal RR intervals; TINN Interpolating triangular histogram of RR intervals; RMSSD Square root of the mean squared differences between adjacent normal RR intervals; un Normalized unit

compared with healthy individuals, suggesting a reduction in the parasympathetic modulation activity. Similar results were also observed in patients with DM1 $\geq 40$ years of age $(8,18)$, and in young subjects with a mean age of 22 years (34).

However, Hägglund et al (33) found no statistically significant differences for the SD1 index in young adults with DM1 at rest. The 
authors suggested this may be related to the small sample size used in the study or the fact that individuals analyzed were under adequate control and treatment of blood glucose, which could mask the alterations generated by diabetes.

Regarding the SD1/SD2 ratio, no statistically significant difference for the DM1 group compared with the control group was observed, which could be explained by the reduction of both SD1 and SD2 indexes.

In the Poincaré plot qualitative analysis, which allows a nonlinear assessment of ANS $(16,33,35)$, was observed a less dispersion of points for the DM1 group compared with the control group. This low dispersion pattern of RR reflects a reduction in HRV, indicating loss of ANS complexity for these individuals (16). In the control group, the visual analysis of Poincaré plot showed a greater beat-to-beat dispersion and long-term RR dispersion which is representative of a normal ANS behaviour $(10,16)$. Similar results were found in studies that also qualitatively analyzed the Poincaré plot in individuals with DM1 $(8,18)$.

All observed findings of geometric indexes were confirmed by the analysis of HRV indexes obtained through the time and frequency domains. Regarding the ANS global activity the SDNN index, which represents the joint action of the sympathetic and parasympathetic activity (10), was lower in the diabetic group compared with the control group, indicating decreased global HRV.

The RMSSD and HF $\mathrm{ms}^{2}$ indexes that represent parasympathetic modulation activity also showed a reduction for DM1 group compared with the control group. Similarly, a reduction in parasympathetic modulation in patients with DM1 (5) and DM2 $(9,36,37)$ have been observed. This reduction is described in the literature as the first sign of CAN (32), which is a strong negative prognosis in relation to allcause mortality in a diabetic population $(38,39)$.

The analysis of the LF $\mathrm{ms}^{2}$ index allowed to observe the sympathetic modulation activity (10), and showed a significant decrease in the DM1 group compared with healthy subjects, indicating a reduction of the sympathetic modulation activity, corroborating with findings in the literature $(40,41)$. However, another study did not find similar results compared with the present one for LF $\mathrm{ms}^{2}$ index (42), which can be due to exogenous insulin increasing sympathetic activity, displacing the balance to the LF component (42), or by the methodological limitations for evaluation this sympathetic activity, because the LF index do not demonstrate a pure activity of this ANS component (43).

Data expressed in standard units contribute to evaluate the sympathovagal balance (44) because they represent the activity of both branches proportionally, with the percentage of each giving a total of $100 \%$. The LF and HF indexes in normalized units showed no significant difference, because there was a decrease in both sympathetic and parasympathetic modulation activity.

One of the findings of the present study stands out by suggesting the changes observed in the branches of diabetic volunteers ANS were found in a condition of absence of clinical complications arising from diabetes.

Indexes obtained by geometrical methods showed excellent correlations with time and frequency domain indexes, indicating that the geometrical indexes are sensitive to identify changes in autonomic modulation.

Especially noteworthy was the excellent correlations between the geometric indexes and the SDNN and RMSSD indexes, obtained from time domain. The SD1 index showed an absolute correlation with RMSSD index and an excellent correlation with HF ms ${ }^{2}$ index, highlighting that these indexes reflect parasympathetic modulation active. A similar result were found for indexes that assess the global HRV, i.e. the SD2, RRtri and TINN indexes showed an excellent correlation with the SDNN index. These correlations appear to be related to the fact that the indexes express the same ANS condition $(45,46)$.

The limitations of the present study should be acknowledged. The cross-sectional design of th epresent study makes it exceedingly difficult to longitudinally analyze the influence of DM1 on ANS; therefore, it could not be concluded that the changes observed in diabetic
ANS are in the initial, intermediate or final stage of the influence by a condition of hyperglycemia on the ANS. Additionally, the diagnosis time of DM1 were different between the participants and the presence of obesity for an individual from each study group, despite both being classified in the same degree of obesity (ie, grade I), may also be considered a limitation of the present study.

Because autonomic dysfunction can affect individuals with DM1, it is important to restore the ANS performance in this population to avoid the complications. The present study provides a new way of interpreting the influence of DM1 on ANS, especially in relation to the qualitative analysis of Poincaré plot, which enables a more representative analysis of the physiological system condition. In addition, the findings of the present study could help in understanding the changes that DM1 in SNA promotes in young individuals and can also contribute to interventions that can be more effective and targeted to the SNA.

\section{CONCLUSIONS}

The results suggest that individuals with DM1 experience a reduction of parasympathetic and sympathetic activities of the ANS, which was observed by geometric and time and frequency indexes. In addition, it was able to verify correlations between geometric indexes and the time and frequency indexes.

ACKNOWLEDGMENTS: The authors are grateful to CNPq (National Council of Scientific and Technological Development - Protocol $n^{\circ}$ 477442/2012-9) and FAPESP (research support foundation of São Paulo state - Process n⿳0 2013/19055) for financial support for this study.

\section{REFERENCES}

1. Vinik AL, Ziegler D. Diabetic cardiovascular autonomic neuropathy. Circulation 2007;115:387-97.

2. Oliveira AF, Valente JG, Leite IC, et al. Global burden of disease attributable to diabetes mellitus in Brazil. Cad Saude Publica 2009;25:1234-44.

3. Diabetes Association. Diagnosis and classification of diabetes mellitus. Diabetes Care 2010;33:S62-S9.

4. Mogensen UM, Jensen T, Kober L, et al. Cardiovascular autonomic neuropathy and subclinical cardiovascular disease in normoalbuminuric type 1 diabetic patients.

Diabetes 2012;61:1822-30.

5. Dungan KM, Osei K, Sagrilla C, et al. Effect of the approach to insulin therapy on glycaemic fluctuations and autonomic tone in hospitalized patients with diabetes. Diabetes Obes Metab 2013;15:558-63.

6. Vitor ALR, Souza NM, Lorenconi R, et al. Nonlinear methods of heart rate variability analysis in diabetes. HealthMed 2012;6:2647-53.

7. Orlov S, Bril V, Orszag A, et al. Heart rate variability and sensorimotor polyneuropathy in type 1 diabetes. Diabetes Care 2012;35:809-16.

8. Guzik P, Piskorski P, Contreras P, et al. Asymmetrical properties of heart rate variability in type 1 diabetes. Clin Auton Res 2010;20:255-7.

9. Seyd A, Joseph PK, Jacob J, et al. Automated diagnosis of diabetes using heart rate variability signals. J Med Syst 2012;36:1935-41.

10. Vanderlei LCM, Pastre CM, Hoshi RA, et al. Noções básicas de variabilidade da frequência cardíaca e sua aplicabilidade clínica. Rev Bras Cir Cardiovasc 2009;24:205-17.

11. Ferreira LL, Souza NM, Bernardo AFB, et al. Variabilidade da frequência cardíaca como recurso em fisioterapia: Análise de periódicos nacionais. Fisioter Mov 2013;26:25-36.

12. Nolan RP, Barry-Bianchi SM, Mechetiuc AE, et al. Sex-based differences in the association between duration of type 2 diabetes. Diab Vasc Dis Res 2009;6:276-82.

13. Soydan N, Bretzel RG, Fischer B, et al. Reduced capacity of heart rate regulation in response to mild hypoglycemia induced by glibenclamide and physical exercise in type 2 diabetes. Metabolism 2013;62:717-24.

14. Fakhrzadeh H, Yamini-Sharif A, Sharifi F, et al. Cardiac autonomic neuropathy measured by heart rate variability and markers of subclinical atherosclerosis in early type 2 diabetes. ISRN Endocrinol 2012:1-7. 
15. Matei D, Popescu CD, Ignat B, et al. Autonomic dysfunction in type 2 diabetes mellitus with and without vascular dementia. J Neurol Sci 2013;325:6-9.

16. Hsu CH, Tsai MY, Huang GS, et al. Poincaré plot indexes of heart rate variability detect dynamic autonomic modulation during general anesthesia induction. Acta Anaesthesiol Taiwan 2012;50:12-8.

17. Vanderlei LCM, Pastre CM, Júnior IFF, et al. Índices Geométricos de Variabilidade da Frequência Cardíaca em Crianças Obesas e Eutróficas. Arq Bras Cardiol 2010;95:35-40.

18. Contreras P, Canetti R, Migliaro E, et al. Correlations between frequency-domain HRV indices and lagged Poincaré plot width in healthy and diabetic subjects. Physiol Meas 2007;28:85-94.

19. Ziegler D, Piolot R, Straburger K, et al. Normal ranges and reproducibility of statistical, geometric, frenquency domain, and non-linear measures of 24-hour heart rate variability. Horm Metab Res 1999:31:672-9.

20. Hallal P, Gomez L, Parra D, et al. Lições aprendidas depois de 10 anos de uso do IPAQ no Brasil e Colômbia. J Phys Act Health 2010;7:S259-S264.

21. Abeso. Diretrizes brasileiras de obesidade - Associação brasileira para o estudo da obesidade e síndrome metabólica. 2009;3:1-83.

22. Ferreira MG, Valente JG, Gonçalves RMVS, et al. Accuracy of waist circumference and waist-to-hip ratio as predictors of dyslipidemia in a cross-sectional study among blood donors in Cuiabá, Mato Grosso State, Brazil. Cad Saude Publica 2006;22:307-14

23. Sociedade Brasileira de Cardiologia. VI Diretrizes brasileiras de hipertensão. Arq Bras Cardiol 2010:95:1-51.

24. Vanderlei LCM, Silva RA, Pastre CM, et al. Comparison of the Polar S810i monitor and the ECG for the analysis of heart rate variability in the time and frequency domains. Braz J Med Biol Res 2008;41:854-9.

25. Gamelin FX, Berthoin S, Bosquet L, et al. Validity of the polar S810 heart rate monitor to measure R-R intervals at rest. Med Sci Sports Exerc 2006;38:887-93.

26. Vanderlei FM, Rossi RC, Souza NM, et al. Heart rate variability in healthy adolescents at rest. J. Hum Growth Dev 2012;22:173-8.

27. Task Force of the European Society of Cardiology and the North American Society of Pacing and Electrophysiology. Heart rate variability: Standards of measurement, physiological interpretation, and clinical use. Circulation 1996;93:1043-65.

28. Carvalho TD, Pastre CM, Rossi RC, et al. Índices geométricos de variabilidade da frequência cardíaca na doença pulmonar obstrutiva crônica. Rev Port Pneumol. 2011;17:260-5.

29. Tulppo MP, MaKikallio TH, Seppanen T, et al. Heart rate dynamics during accentuated sympathovagal interaction. Am J Physiol Heart Circ Physiol 1998;274:H810-H816.

30. Niskanen JP, Tarvainen MP, Ranta-aho PO, et al. Software for advanced HRV analysis. Comput Methods Programs Biomed 2004;76:73-81.
31. Hoshi R, Pastre C, Vanderlei LCM, et al. Poincaré plot indexes of heart rate variability: Relationships with other nonlinear variables. Auton Neurosci 2013;177:271-4.

32. May O, Arildsen H. Long-term predictive power of heart rate variability on all-cause mortality in the diabetic population. Acta Diabetol 2011;48:55-9.

33. Hägglund H, Uusitalo A, Peltonen JE, et al. Cardiovascular autonomic nervous system function and aerobic capacity in type 1 diabetes. Front Physiol 2012;3:356.

34. Javorka M, Javorková J, Tonhajzerová I, et al. Heart ratjje variability in young patients with diabetes mellitus and healthy subjects explored by Poincaré and sequence plots. Clin Physiol Funct Imaging 2005;25:119-27.

35. Khandoker A, Palaniswami M, Begg R, et al. A comparative study on approximate entropy measure and poincare plot indexes of minimum foot clearance variability in the elderly during walking. J Neuroeng Rehabil 2008;5:1-10.

36. Javorka M, Trunkvalterova Z, Tonhajzerova I, et al. Short-term heart rate complexity is reduced in patients with type 1 diabetes mellitus. Clin Neurophysiol 2008;119:1071-81.

37. Skinner JE, Weiss DN, Anchin JM, et al. Nonlinear PD2i heart rate complexity algorithm detects autonomic neuropathy in patients with type 1 diabetes mellitus. Clin Neurophysiol 2011;122:1457-62.

38. Xhyheri B, Manfrini O, Mazzolini M, et al. Heart rate variability today. Prog Cardiovas Dis 2012;55:321-31.

39. Rodrigues TC, Ehrlich J, Hunter CM, et al. Reduced heart rate variability predicts progression of coronary artery calcification in adults with type 1 diabetes and controls without diabetes. Diabetes Technol Ther 2010;12:963-9.

40. Sucharita S, Ganapathi B, Jyothi I, et al. Autonomic nervous system function in type 2 diabetes using conventional clinical autonomic tests, heart rate and blood pressure variability measures. Indian J Endocrinol Metabo. 2011;15:198-203.

41. Traon AP, Fontaine S, Tap G, et al. Cardiovascular autonomic neuropathy and other complications in type 1 diabetes. Clin Auton Res 2010;20:153-60.

42. Lucini D, Zuccotti G, Malacarne M, et al. Early progression of the autonomic dysfunction observed in pediatric type 1 diabetes mellitus. Hypertension. 2009;54:987-94.

43. Souza NM, Rossi RC, Vanderlei FM, et al. Heart rate variability in obese children. J Hum Growth Dev. 2012;22:328-33.

44. Chun MY, Park HK, Hwang HS, et al. The association between symptoms of autonomic neuropathy and the heart rate variability in diabetics. Korean J Fam Med 2011;32:292-8.

45. Brennan M, Palaniswami M, Kamen P, et al. Do existing measures of Poincare plot geometry reflect nonlinear features of heart rate variability? IEEE Trans Neural Syst Rehabil Eng 2011;48:1342-7.

46. Karmakar C, Khandoker A, Gubbi J, et al. Complex correlation measure: a novel descriptor for Poincaré plot. Biomed Eng Online 2009;8:1-12. 